Short report

\title{
Dyspnoea during generalised spasms in tetraplegic patients
}

\author{
JR SILVER, RP LEHR \\ From the National Spinal Injuries Centre, Stoke Mandeville Hospital, Aylesbury, England, and \\ the Department of Anatomy, School of Medicine, Southern Illinois University, Carbondale, Illinois, USA
}

SUMMARY Dyspnoea during generalised spasms is reported in three tetraplegic patients. Intraoesophageal electromyographic evidence strongly suggests that the dyspnoea is a result of diaphragmatic spasms. Utilisation of this EMG technique in other tetraplegic patients may add to our knowledge of variations in the distribution of the phrenic nerve nucleus within the spinal cord.

Our purpose is to focus attention on the occurrence of a previously unreported phenomenon, dyspnoea during generalised spasms in tetraplegic patients. Thirteen patients with complete or incomplete spinal injuries ranging from $\mathrm{C} 4$ to $\mathrm{T} 6$ as the last intact segmental level have had an intra-oesophageal electromyographic electrode passed as part of an investigation into their ventilatory mechanics. During these ongoing investigations, inquiry was made about breathing during generalised spasms. Three patients reported dyspnoea during a generalised spasm. This phenomenon was not present immediately after injury when the patient was in spinal shock. It was found during a generalised muscular spasm; the earliest time it was elicited was some six weeks after injury and was accompanied by a sensation of breathlessness. We suggest that this phenomenon is a result of a spasm of the diaphragm. Previous reports concerning the function of the diaphragm in patients with neurological disorders have been limited to studies on patients with a complete loss of one phrenic nerve, resulting in hemidiaphragm or of bilateral diaphragmatic paralysis. ${ }^{1-3}$

\section{Patients}

\section{Case 1}

A thirty-year-old male suffered fractures of his fourth and fifth cervical vertebrae during a rugby match and was examined six years after injury. He had normal cranial

Address for reprint requests: Dr JR Silver, National Spinal Injuries Centre, Stoke Mandeville Hospital, Aylesbury HP21 8AL, UK.

Accepted 1 July 1981 nerves and power in the sternocleidomastoid and trapezius, weak power in the deltoid and biceps muscles and no power below this level. The last normal segment of sensation was C5; there was no sensation below this level. His vital capacity was 1.9 litres.

Case 2

A fifty-year-old male was examined eight years after injury. He had sustained a fracture of his sixth cervical vertebra in a fall down stairs. He had normal cranial nerves with weakness of the deltoid and biceps; there was no power below this level. Sensory loss was complete below the fifth cervical segment. His vital capacity was $1 \cdot 6$ litres.

\section{Case 3}

A sixty-three-year-old male sustained a dislocation of his third upon fourth cervical vertebrae giving rise to incomplete tetraplegia with weakness of both deltoid muscles as the result of a motor cycle accident. He was examined one month after injury. Initially there was no power at all in his upper extremities, but over the ensuing months, power returned to his right upper limb and both lower limbs. The last normal level of sensation was $\mathrm{C} 4$. Radiographic screening of the diaphragm was normal. Vital capacity was 1.8 litres.

\section{Techniques}

The patients were investigated electromyographically using principally bipolar fine-wire electrodes ${ }^{4}$ for the intercostal muscles. An intra-oesophageal electromyographic electrode ${ }^{5}$ was used to record the diaphragmatic EMG. The combination of intra-oesophageal and bipolar fine-wire electrodes allows the differentiation of the diaphragmatic and intercostal EMG. Simultaneous spirometry was carried out. (For a fuller description of the technique, see Silver and Lehr. $\left.{ }^{6}\right)$ 


\section{Observations}

The three patients described the dyspnoea in their chest as uncomfortable, but not painful, being a tightness below the ribs, as though someone was sitting on the chest, or as an explosion of air within their chest. The dyspnoea was a continuation of generalised body spasms which were initiated by sudden movements such as having their position changed, when they received passive movements to the arms or legs, or as part of another reflex, for example plantar stimulation. The common pattern of general spasm of the whole body was clenching of the fingers, flexion of the elbows, adduction of the shoulder or extension of the whole arm with extension of the fingers; whether the arms were flexed or extended the lower limbs were involved, also either flexing or extending. The most common method of producing a generalised spasm was by taking a deep breath. Voluntary breathing was not possible during the chest component of the generalised spasm nor could the dyspnoea be stopped voluntarily. Onset of the dyspnoea always followed the initiation of a general spasm and was subject to the same conditions which influence the onset of the general spasm. These are not present during spinal shock but appear at about six weeks when spasticity develops in the voluntary muscles. A generalised spasm is more likely to occur when the patient has been maintained in the same position for a prolonged period, in a colder environment or if anxious about a situation. Spasms are reflex in nature and adapt; thus once a spasm has occurred subsequent spasms are more difficult to elicit and of decreased intensity. The duration of the dyspnoea following a generalised spasm was variable both between spasms in an individual patient and between patients.

We concluded that the dyspnoea was a spasm of the diaphragm based on EMG activity, spirometric activity and clinical observations (fig 1a). The beginning of the record shows normal ventilation in the patient, evidenced by the spirometry trace. (a) A deep breath with an increasing contraction of the diaphragm is noted, which is interrupted by a brief pause during expiration. Diaphragmatic EMG activity indicates two inspirations as preparation for coughs following which the patient was requested to strain by trying to expire against a closed glottis. It should be noted in the diaphragmatic lead that the down-going after-spike discharges ${ }^{7}$ are abnormal. (b) Upon inspiration to a deep breath the beginning of a generalised spasm is initiated. The spirometric trace shows that the breath is held in inspiration, expiration is not begun until the spasm of the diaphragm ceases. The normal activity of the diaphragm ceases and an abnormal EMG interference pattern of increased amplitude is apparent. Concurrent EMG activity is noted in the seventh intercostal space EMG trace. It should be noted that onset of activity in the three EMG channels is not synchronised.

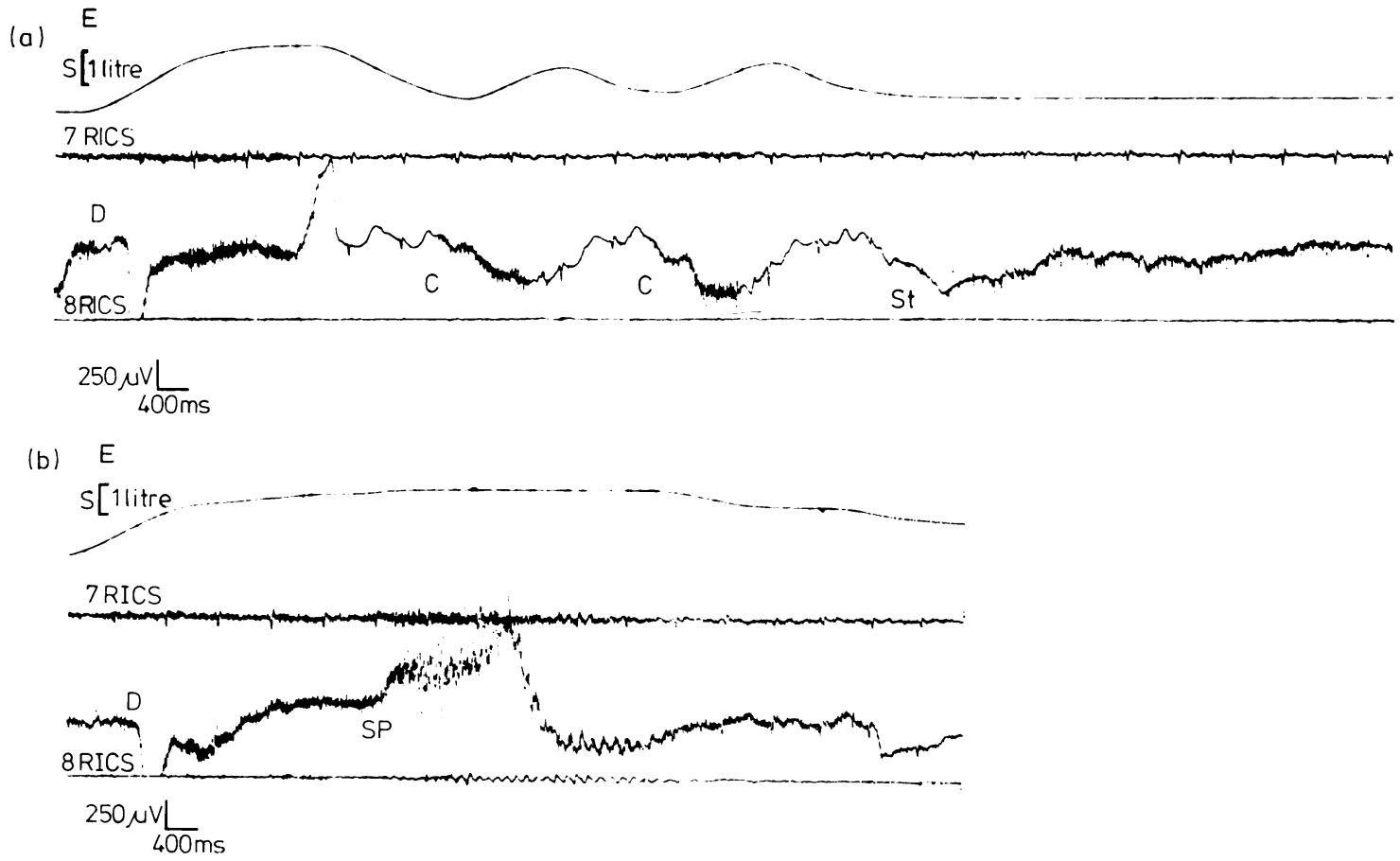

Fig (a) Channel I-S, spirometer trace; Channel 2-7RICS-seventh right intercostal space, wire electrode;

Channel 3-D. diaphragm; Channel 4-8RICS-eighth right intercostal space. C-cough. St-strain. Sp-spasm.

The trace is continuous. (b) Note the spasm of the diaphragm in trace $b$. 
Detailed calculations of the duration of the diaphragmatic EMGs for five spasms in Patient 1 provide a mean of 8.7 seconds with a range of 3.5 to $12 \cdot 1$ seconds.

\section{Discussion}

Generalised spasms were described by Head and Riddoch $^{8}$ during the First World War; they appeared some four weeks after spinal injury. "It is evident, therefore, that under certain conditions the spinal cord below the level of the lesion may show signs of diffuse reflex activity. Scratching the sole of the foot may not only evoke a flexor spasm, but may cause premature evacuation of the bladder and an outburst of excessive sweating. This we have spoken of as a 'mass-reflex'." In the same paper they described this reflex causing contraction of the abdominal muscles. When we observed dyspnoea during a spasm in patients with spinal cord injuries we originally attributed this dyspnoea to the abdominal muscles contracting as part of the mass reflex and pushing the abdominal contents upwards into the diaphragm, thus mechanically impeding its action. We do not believe this to be the case. We have not seen this particular form of dyspnoea, except in patients with C5 lesions. The other ten patients did not show it. Although clinically the abdominal muscles appeared to contract during the spasms and previously we have elicited, with difficulty, electromyographic activity in the abdominal muscles, during these investigations, using more sensitive equipment and bipolar fine-wire electrodes, we were not able to do so.

The present spirometric, electromyographic and clinical observations suggest that the generalised spasm involves the diaphragm. All three patients had reduced vital capacities. Other studies ${ }^{9}$ have shown that the vital capacity in tetraplegic patients is about half a litre, when it indicates partial paralysis of the diaphragm. It seems in these patients that the diaphragm was partially paralysed from the outset and remained so.

Spasticity is a reflex phenomenon, characteristic of muscles in which the innervation is intact but there is partial or complete paralysis as a result of physiological or anatomical separation from supraspinal control. Michaelis ${ }^{10}$ points out that hypertonus of basic spasticity due to the lesion of the spinal cord itself may be stimulated into a spasm by superimposing additional aberrant stimuli to the widened receptive field. The widened receptive field may be triggered by stimulation of the cutaneous afferents of touch, the temperature afferents or by the proprioceptive afferents of the tendon organs or muscle spindles. The spasm may be mild and localised to one extremity or it may be generalised and spread to the trunk and other limbs. The spasm is due to radiation of stimuli at a cord level in the partially damaged spinal motor neurones; this involves the phrenic nucleus. Classically, the phrenic nerve nucleus is placed at cervical cord levels 3,4 and 5 and is supposed to have one of the most consistent locations in the cord. Accessory phrenic nerve innervation to the diaphragm has been proposed as well as reports of innervation from the ansa cervicalis. ${ }^{11}$ The presence of the spasms in the diaphragm of these patients suggests a partial destruction of the phrenic nerve nucleus. This would place a portion of the nucleus as low as cervical segment six. The work of Keswani and Hollinshead would support this contention based on histological examination of cords from ten patients who had undergone phrenicotomy. ${ }^{12}$ In the cords of the phrenicotomy patients, chromatolysed cells were identified at the level of the sixth cervical segment. We suggest that these patients with the spasm in the diaphragm demonstrate a portion of the phrenic nucleus to be at least as far caudal as $\mathbf{C 6}$. The separation of the more caudal segments below the injury from the supraspinal centres enable the lower motor neurones of the phrenic nucleus to respond to the local stimulation and produce a diaphragmatic spasm.

It is striking that the only patients that showed the full evolution of spasms to involve the shoulders and diaphragm were those patients in whom the deltoid was partially paralysed. Embryologically, the diaphragm receives innervation from the $3 \mathrm{rd}, 4 \mathrm{th}$, 5th and perhaps 6th myotomes, one or more of which will certainly supply the deltoid and thus serve as a marked indicator when the diaphragm is involved.

The importance of this report is the recognition of this previously unreported phenomenon and the possibility it offers for a novel approach to a clarification of the cord levels of the human phrenic muscles. This may require development of a directional intra-oesophageal electrode, but the resultant information could add to our understanding of the motor cell distribution in the human spinal cord.

\section{References}

${ }^{1}$ Newsom Davis J, Goldman M, Loh L, Casson M. Diaphragm function and alveolar hypoventilation. Quart J Med 1976;45:87-100.

${ }^{2}$ Higenbottom T, Allen D, Loh L, Clark TJH. Abdominal wall movement in normals and patients with hemidiaphragmatic and bilateral diaphragmatic palsy. Thorax 1977;32:589-95.

${ }^{3}$ Claque HW. Effects of posture on lung volume: Airway closure and gas exchange in hemidiaphragmatic paralysis. Thorax 1979;34:523-6.

4 Basmajian JV. Muscles alive: their functions revealed by electromyography. 4th ed. Baltimore: Williams and 
Wilkins, 1978:1037.

"Schweitzer TW, Fitzgerald JW, Bowden JA, LynneDavies P. Spectral analysis of human inspiratory diaphragmatic electromyograms. $J$ Appl Physiol 1979;46:152-65.

' Silver JR, Lehr RP. Electromyographic investigation of the diaphragm and intercostal muscles in tetraplegics. J Neurol Neurosurg Psychiatry. 1981;44: 837-42.

' Silver JR, Abdel-Halim RE. Chest movements and electromyography of the intercostal muscles in tetraplegic patients. Paraplegia 1971 ;9:123-31.

${ }^{8}$ Head H, Riddoch G. The automatic bladder, excessive sweating and some other reflex conditions in gross injuries of the spinal cord. Brain 1917;40:188-263.
${ }^{9}$ Guttmann L, Silver JR. Electromyographic studies on reflex activity of the intercostal and abdominal muscles in cervical cord lesion. Paraplegia 1965;3: $1-22$.

${ }^{10}$ Michaelis LS. Spasticity in spinal cord injuries. In: Vinken PJ, Bruyn GW. Injuries of the spine and spinal cord. Part II. New York: American Elsevier Co, 1976:477-87.

11 Warwick R, Williams PL. Gray's Anatomy, 35th British edition. Philadelphia: WB Saunders Co, 1973:1037.

12 Kewswani NH, Hollinshead WH. Localisation of the phrenic nucleus in the spinal cord of man. Ant Rec 1956;125:683-700. 